\title{
Identification, Characterization, and In Silico Analysis of New Imine Reductases From Native Streptomyces Genomes
}

\begin{abstract}
César Iglesias ${ }^{1,2}$, Ariel Tijman ${ }^{1,2}$, Gonzalo López ${ }^{1,2}$, Maria Inés Lapaz ${ }^{1}$, Maria Julia Pianzzola ${ }^{1}$ Paola Panizza ${ }^{1,2 *}$ and Sonia Rodríguez Giordano ${ }^{1,2 *}$

${ }^{1}$ Molecular Microbiology Lab, Bioscience Department, Facultad de Química, Universidad de la República(Udelar), Montevideo, Uruguay, ${ }^{2}$ Biocatalysis and Biotransformation Lab, Organic Chemistry Department and Bioscience Department, Facultad de Química, Universidad de la República(Udelar), Montevideo, Uruguay
\end{abstract}

OPEN ACCESS

Edited by:

Fabricio R. Bisogno,

CONICET Instituto de Investigaciones

en Físico Química de Córdoba

(INFIQC), Argentina

Reviewed by:

Juan Mangas-Sanchez, Instituto de Síntesis Química y Catálisis Homogénea (ISQCH), Spain José Luis Borioni, Universidad Nacional de Cuyo, Argentina

*Correspondence: Paola Panizza ppanizza@fq.edu.uy Sonia Rodríguez Giordano soniar@fq.edu.uy

Specialty section: This article was submitted to Biocatalysis,

a section of the journal Frontiers in Catalysis

Received: 29 September 2021 Accepted: 25 October 2021 Published: 25 November 2021

Citation:

Iglesias C, Tijman A, López G, Lapaz MI, Pianzzola MJ, Panizza P and Rodríguez Giordano S (2021) Identification, Characterization, and In

Silico Analysis of New Imine Reductases From Native Streptomyces Genomes.

Front. Catal. 1:785963.

doi: 10.3389/fctls.2021.785963
The development of biocatalytic tools for the synthesis of optically pure amines has been the focus of abundant research in recent years. Among other enzymes, imine reductases have attracted much attention associated with the possibility of attaining chiral secondary amines. Furthermore, the reductive aminase activity associated with some of these enzymes has facilitated the production of optically pure amines from a prochiral ketone, a transformation that opens doors to an incredible array of products. In this work, the genomes from native Streptomyces strains isolated in our lab have been explored on the search for novel imine reductases. Application of different structural criteria and sequence motif filters allowed the identification of two novel enzymes, SsIRED_S and Ss-IRED_R. While the former presented outstanding activity towards bulky cyclic imine substrates, the latter presented reductive aminase activity with the assayed ketones. A bioinformatic analysis based on modeling and docking studies was performed in order to explain the differences in enzyme activity, searching for additional criteria that could be used to analyze enzyme candidates in silico, providing additional tools for enzyme selection for a particular application. Our findings suggest that imine reductase activity could be predicted by this analysis, overall accounting for the number of docking positions that meet the catalytic requirements.

Keywords: imine reductases, reductive aminases, Streptomyces, docking, bioinformatic analysis

\section{INTRODUCTION}

Synthesis of chiral amines is of particular interest for synthetic organic chemistry due to its presence in many biologically active molecules. Different methodologies and strategies have been developed for amine synthesis, and the biocatalytic toolbox of enzymes (i.e., transaminases, amine dehydrogenases, monoamine oxidases, and imine reductases (IREDs)) has become of interest to prepare these compounds in a more effective and stereoselective manner (France et al., 2017; Cosgrove et al., 2018; Devine et al., 2018; Höhne 2019; Adams et al., 2019).

IREDs are NADPH-dependent oxidoreductases able to catalyze the asymmetric reduction of different imines and iminium ions to produce the corresponding secondary and tertiary amines (Cosgrove et al., 2018; Höhne 2019). These enzymes can be found in different biosynthetic pathways 
and were discovered by Mitsukura et al. (2011) in Streptomyces sp. (Mitsukura et al., 2013). Over the last years, several reports of IREDs have been published; noticeably, gene selection was entirely based on sequence analysis by alignments (France et al., 2017; Lenz et al., 2017; Matzel, Gand, and Höhne 2017; Devine et al., 2018; Höhne 2019; Montgomery et al., 2020; Yao et al., 2021). In 2017, an IRED homologue capable of performing reductive amination (RedAm) was found in Aspergillus oryzae (Aleku et al., 2017). IRED and RedAm activities are similar, but their mechanisms are slightly different (Rodríguez-Mata et al., 2013; Man et al., 2015; Sharma et al., 2018). Due to the convenience of this reaction, which allows the synthesis of secondary amines directly from ketones, efforts have been made to expand the repertoire of enzymes capable of performing reductive amination (Kohls, Steffen-Munsberg, and Höhne 2014; Schrittwieser, Velikogne, and Kroutil 2015; Wetzl et al., 2016; Aleku et al., 2017; Maugeri and Rother 2017; France et al., 2018). An interesting article published by Turner et al. made a characterization of 80 putative and 15 previously described IREDs across 10 different transformations and confirmed that reductive amination catalysis is not limited to any particular subgroup or sequence motif (Montgomery et al., 2020).

Nowadays, most of IRED and RedAm are not suitable for industrial applications mainly due to their limited substrate scope, low activity towards bulky substrates, the need of an excess of the amine nucleophile, and enzyme instability under process conditions. Nevertheless, there are a few recent examples of success in the application of these enzymes to industrial processes, all of which required a previous improvement of the enzymes' performance using intensive and process focused enzyme engineering (Schober et al., 2019; Duan et al., 2021; Kumar et al., 2021). Other protein engineering approaches have been explored to overcome the limitations, and different biocatalysts have already been used in preparative scale biotransformations and enzymatic cascade reactions (France et al., 2017; Lenz et al., 2017; Matzel, Gand, and Höhne 2017; Devine et al., 2018; Höhne 2019; Yao et al., 2021). A deeper understanding of structure-activity relationship for these enzymes is needed for further improvement, as well as the development of alternative ways for uncovering new enzymes.

As sequence databases are growing exponentially, enzyme discovery using bioinformatic approaches is increasingly appealing. Through sequence alignments, in silico studies of proteins allow the recognition of conserved motifs among different enzymes of the same family, providing an interesting way to explore new proteins for a desired activity and to get deeper insights into sequence-function relationships (Höhne et al., 2010; Iglesias, Panizza, and Rodriguez 2017). Provided that adequate templates are available, homology modeling can be used to build proper models of protein structures from its sequence (Catucci et al., 2016; Fademrecht et al., 2016; Velikogne et al., 2018). Moreover, de novo accurate prediction of protein structure is becoming feasible (Senior et al., 2020; 2019). Docking experiments can be of major help in predicting substrate binding and understanding the role of different amino acids present in the active site (Bommarius, Blum, and Abrahamson 2011; Sirin et al., 2014; Catucci et al., 2016; Han et al., 2017; Montgomery et al., 2020). Deeper in silico analysis involves the use of quantum mechanics/molecular mechanics approach, but the high computational cost of these methods is often a limitation for its application (Rinaldi et al., 2018). There are already some reports on in silico studies for IREDs, and specific databases of their sequences have been constructed (Fademrecht et al., 2016; Velikogne et al., 2018).

As already mentioned, the first IREDs were identified from Streptomyces strains. This genus has been largely known for its diversity on secondary metabolites; thus, the probability of finding novel IREDs among strains of this genus is large (Spasic et al., 2018). In our lab, Prof. Pianzzola's group has built a collection of over 200 Streptomyces strains isolated and characterized from potato tubers and soil samples from Uruguay (Lapaz et al., 2017; Croce et al., 2021). Recently, the whole-genome sequencing of the representative strains of this collection was reported (Lapaz et al., 2019). In this work, we decided to explore the available genomes from this Uruguayan Streptomyces collection on the search for novel enzymes with IRED or RedAm activity. Two new IRED candidates with potential IRED or reductive aminase activity were selected based on structural motifs. Furthermore, in silico analysis using homology models refined by molecular dynamics simulation and docking studies proved useful to explain the catalytic activity of these enzymes and could provide a valuable tool to select novel enzymes with IRED or RedAm activity.

\section{MATERIALS AND METHODS}

\section{Strains and Growth Conditions}

Escherichia coli BL21(DE3) was acquired from New England BioLabs (Ipswich, MA, USA) and was cultivated in Luria-Bertani (LB) broth or on LB agar plates at $37^{\circ} \mathrm{C}$. Recombinant strains E. coli BL21(DE3) (pET-28b(+)-SsIRED_R) and E. coli BL21(DE3) (pET-28b(+)-Ss-IRED_S) were grown in LB broth or LB agar plates, supplemented with the appropriate antibiotic (kanamycin $50 \mu \mathrm{g} / \mathrm{ml}$ ). Autoinduction media LB Base including trace elements was used for Ss-IRED_R and Ss-IRED_S protein production. Recombinant strains of E. coli BL21(DE3) coexpressing an IRED and $L b-\mathrm{ADH}$ required a second antibiotic (ampicillin $50 \mu \mathrm{g} / \mathrm{ml}$ ) as well anhydrotetracycline $(200 \mu \mathrm{g} / \mathrm{ml})$ for protein production.

Ss-IRED_R (National Center for Biotechnology Information (NCBI) ref. seq. WP_112474446.1) was identified from Streptomyces sp. strain ST1020 (BioSample: SAMN04977949). Ss-IRED_S (NCBI ref. seq. WP_119590418.1) was identified from Streptomyces scabies strain ST129 (BioSample: SAMN07731257).

\section{Chemicals and Molecular Biology Reagents}

Biotransformation substrate 1a was kindly provided by Prof. Ignacio Carrera; substrates $\mathbf{1 b}$-1 d were generously donated by Prof. Nicholas Turner; substrates 3-5 and a were purchased from Sigma Aldrich (St Louis, MO, USA). All the biotransformation substrates were used without further purification. Racemic amines used as analytical standards 
were purchased from Sigma Aldrich (St. Louis, MO, USA), while chiral amines were produced from the prochiral imines or ketones through biotransformations with reported biocatalysts: 2a was produced with IRED-K; 2b was produced with IRED-D and K (Velikogne et al., 2018); 2c was produced with Ao-IRED (Aleku et al., 2016) and GF3546IRED (Mitsukura et al., 2013); and 4a was produced with AspRedAm (Aleku et al., 2017).

Molecular biology reagents were purchased from New England Biolabs (Ipswich, MA, USA) and Thermo Fisher Scientific (Waltham, MA, USA). Expression vector pET-28b(+) was purchased from Novagen (Darmstadt, Germany) and was used for gene expression. Fourteen pET-28a(+) IRED-containing plasmids (IRED-A to IRED-N) and plasmid pASK-IBA5plus- $L b$ $\mathrm{ADH}$ were very generously donated by Dr. Jörg H. Schrittwieser (Velikogne et al., 2018).

\section{DNA Manipulation and Ss-IRED_S Cloning}

Standard procedures were used for DNA manipulation, and genomic DNA from Streptomyces was extracted according to Sambrook Molecular Cloning: A Laboratory Manual (Sambrook 2001). Plasmid DNA was purified using commercial chromatography kits from Thermo Fisher Scientific (Waltham, MA, USA) and QIAGEN (Hilden, Germany); silica columns from these kits were regenerated and reused following the procedure described by Siddappa (Siddappa et al., 2007), while standard protocols were modified to obtain greater yields of DNA (Pronobis, Deuitch, and Peifer 2016; Wood et al., 2017). Restriction enzymes and thermostable polymerases were used according to the manufacturers' instructions. PCR amplifications were performed in a GeneAMP PCR system 2400 (PerkinElmer, Waltham, MA, USA) using adequate cycling periods, and DNA samples were routinely analyzed by agarose gel electrophoresis (Sambrook 2001). Nucleic acid concentration and purity were measured using a NanoDrop ${ }^{\circledR}$ ND-1000 spectrophotometer. Transformations of electrocompetent cells were performed on a BioRad MicroPulser ${ }^{\mathrm{TM}}$ Electroporator following manufacturer protocols.

An optimized sequence for E. coli of Ss-IRED_R (provisional ID Bankit 2503810) was synthetized and cloned in pET-28b(+) by GenScript (Piscataway, NJ, USA). The resulting vector was transformed into E. coli BL21(DE3) for protein expression, yielding the recombinant strain E. coli BL21(DE3) (pET28b(+)-Ss-IRED_R).

Amplification of Ss-IRED_S was performed by PCR with primers IRED NdeI Fw (GCTCATATGCCCGCACACATC GCAG) and IRED EcoRI Rv (CTAGAATTCGACGAGGGA AACCCCGAC). Ss-IRED_S gene was then cloned into pGEMT Easy vector (Promega, WI, USA), and digestion with NdeI and EcoRI allowed subcloning into pET-28b(+) in frame with the $\mathrm{N}$-terminal His6 tag. The obtained vector was purified by gel electrophoresis using the Freeze ' $\mathrm{N}$ Squeeze kit from Bio-Rad (Hercules, CA, USA) and transformed into E. coli BL21(DE3), yielding the recombinant strain E. coli BL21(DE3) (pET28b(+)-Ss-IRED_S).

\section{Whole Cell Biotransformations With Strains Expressing Ss-IRED_R and Ss-IRED_S}

Fresh plates of E. coli BL21(DE3) (pET-28b(+)-Ss-IRED_R) and E. coli BL21(DE3) (pET-28b(+)-Ss-IRED_S) were streaked from frozen stocks, and a single colony was used to inoculate $5 \mathrm{ml}$ of LB-Kan. The culture was incubated in a rotary shaker overnight $\left(150 \mathrm{rpm}, 37^{\circ} \mathrm{C}\right)$, and $1 \mathrm{ml}$ of this culture was used to inoculate $100 \mathrm{ml}$ of fresh autoinduction media LB Base including trace elements and kanamycin. This culture was incubated in a rotary shaker at $150 \mathrm{rpm}$ for about $2.5 \mathrm{~h}$ at $37^{\circ} \mathrm{C}$ until an $\mathrm{OD}_{600 \mathrm{~nm}} \approx 1$; and then it was grown at $150 \mathrm{rpm}$ and $28^{\circ} \mathrm{C}$ for $48 \mathrm{~h}$. The cells were collected by centrifugation at $4,000 \times g$ and $4^{\circ} \mathrm{C}$ for $15 \mathrm{~min}$. The pellet was washed three times with $50 \mathrm{ml}$ of Tris buffer (100 mM, pH 7.5). E. coli cells measuring $50 \mathrm{mg}$ (wet weight) were resuspended in $465 \mu$ l of the same buffer; glucose and the appropriate substrate were added to a final concentration of $2 \%$ and $5 \mathrm{mM}$, respectively. For RedAm reactions, 50 eq. of propargylamine was used. The reaction mixture was incubated at $28^{\circ} \mathrm{C}$ and $150 \mathrm{rpm}$ for $48 \mathrm{~h}$. Reactions were quenched by addition of $10 \mathrm{M}$ of $\mathrm{NaOH}(100 \mu \mathrm{l})$. The mixture was extracted with ethyl acetate $(750 \mu \mathrm{l})$, and the organic layer was separated by centrifugation $(5 \mathrm{~min}, 12,000 \times g)$. The organic phase was dried with $\mathrm{Na}_{2} \mathrm{SO}_{4}$, and methyl heptadecanoate (2.5 mM) was added as internal standard. Percent conversion and enantiomeric excess were determined as stablished in the Gas Chromatography Analysis section.

\section{Whole Cell Biotransformations With Strains Coexpressing an Imine Reductase and Lb-ADH}

Fresh plates of E. coli BL21(DE3) coexpressing an IRED and $L b$ $\mathrm{ADH}$ were streaked from frozen stocks, and a single colony was used to inoculate $5 \mathrm{ml}$ of LB-Kan + Amp. The culture was incubated in a rotary shaker overnight $\left(150 \mathrm{rpm}, 37^{\circ} \mathrm{C}\right)$, and $1 \mathrm{ml}$ of this culture was used to inoculate $100 \mathrm{ml}$ of fresh autoinduction media LB Base including trace elements and the appropriate antibiotics. This culture was incubated in a rotary shaker at $150 \mathrm{rpm}$ for about $2.5 \mathrm{~h}$ at $37^{\circ} \mathrm{C}$ until an $\mathrm{OD}_{600 \mathrm{~nm}} \approx 1$; $\mathrm{ADH}$ production was induced by the addition of anhydrotetracycline. Then, it was grown at $150 \mathrm{rpm}$ and $28^{\circ} \mathrm{C}$ for $48 \mathrm{~h}$. The cells were collected by centrifugation at $4,000 \times g$ and $4^{\circ} \mathrm{C}$ for $15 \mathrm{~min}$. The pellet was washed three times with $50 \mathrm{ml}$ of Tris buffer (100 mM, pH 7.5). E. coli cells measuring $50 \mathrm{mg}$ (wet weight) were resuspended in $465 \mu \mathrm{l}$ of the same buffer; glucose and the appropriate substrate were added to a final concentration of $2 \%$ and $5 \mathrm{mM}$, respectively. The reaction mixture was incubated at $28^{\circ} \mathrm{C}$ and $150 \mathrm{rpm}$ for $48 \mathrm{~h}$. Reactions were quenched by addition of $10 \mathrm{M}$ of $\mathrm{NaOH}$ $(100 \mu \mathrm{l})$. The mixture was extracted with ethyl acetate $(750 \mu \mathrm{l})$, and the organic layer was separated by centrifugation $(5 \mathrm{~min}$, $12,000 \times g$ ). The organic phase was dried with $\mathrm{Na}_{2} \mathrm{SO}_{4}$, and methyl heptadecanoate $(2.5 \mathrm{mM})$ was added as internal standard. Percent conversion and enantiomeric excess were determined as stablished in the Gas Chromatography Analysis section. 


\section{Gas Chromatography Analysis}

Biotransformation products were analyzed on a GC-2010 Shimadzu (Kyoto, Japan) gas chromatograph equipped with a FID detector. Carrier gas, $\mathrm{H}_{2}$; makeup gas, $\mathrm{N}_{2}$. Columns MEGASE52 (achiral SP, $30 \mathrm{~m} \times 0.25 \mathrm{~mm} \times 0.25 \mu \mathrm{m}$ ) and MEGA-DEX ASX-1 (chiral SP, $25 \mathrm{~m} \times 0.25 \mathrm{~mm} \times 0.25 \mu \mathrm{m}$ ) from MEGA (Milan, Italy) were used for determination of conversion and enantiomeric excess, respectively. Particular analytical conditions and retention times for each amine can be found in Supporting Information, Supplementary Tables S1, S2.

\section{Bioinformatic Analysis}

YASARA's homology modeling experiments, version 21.8.27, were carried out with five templates and three PSI-BLAST iterations in template search; the remaining parameters were set by default (Krieger and Vriend 2014). The templates were initially prepared by adding bond orders and charges; water molecules were removed, and hydrogens were added accounting for protonation states of all ionizable residues at $\mathrm{pH}$ 7.5. IREDs from A. oryzae (PDB 5G6R), A. terreus (PDB 5OJL), Bacillus cereus (PDB 4D3F), and Amycolatopsis orientalis (PDB 5A9T); and RedAm from A. terreus (PDB 6EOD) were used as structural templates for modeling Ss-IRED_R, Ss-IRED_S, IRED-A, B, C, D, F, I, J, K, L, M, and N. To aid alignment correction and loop modeling, a secondary structure prediction for the target sequence was obtained. This was achieved by running PSI-BLAST to create a target sequence profile and feeding it to the PSI-Pred secondary structure prediction algorithm (Jones 1999). A total of 25 independent models were created, and YASARA combined the best parts of the 25 models to obtain a hybrid model. In each homology model using YASARA software, we assigned bond orders and protonation patterns according to $\mathrm{pH} 7.4$ (Krieger et al., 2012).

Refinement by short molecular dynamics simulations was performed to improve structures' quality: with the use of YASARA's force field YAMBER3 (Krieger et al., 2004), 500 ps of molecular dynamics simulation was conducted at $298 \mathrm{~K}$ and $\mathrm{pH}$ 7.4. The simulation was performed in explicit solvent with TIP3P water model, density 0.997 . Based on the lowest energy, the final representative structures were considered for further study. Ligands were first prepared using YASARA's molecular modeling software (Krieger, Koraimann, and Vriend 2002). Solvation of the ligands prior to minimization was carried out in an explicit solvent shell at $\mathrm{pH} 7.4$ and $0.9 \% \mathrm{NaCl}$. Each ligand was subjected to energy minimization to refine the bond lengths and bond angles. The minimized conformation with the lowest energy for each ligand was used in the docking experiments. Docking was performed by VINA software (Trott and Olson 2019) using default parameters. However, the number of VINA runs was increased from 10 to 250 . Size of the docking box was set to $5 \AA$ around the amino acids in the active site in each structure. Positions with a root mean square deviation (RMSD) lower than $2.5 \AA$ were clustered together, and each cluster was represented by the position with lowest free energy of binding. Every cluster obtained by VINA for each substrate used in imine reduction was analyzed, and parameters such as energy of binding, distance for hydride transfer from $\mathrm{NADPH}$ to $\mathrm{C}=\mathrm{N}$ double bond, and interactions between nitrogen of imine with surrounding possible proton donor amino acids His, Glu, Asp, Ser, Tyr, and Cys were evaluated.

\section{RESULTS}

\section{Sequence Alignments and Analysis of Primary Structure}

We used BLASTp of NCBI (Altschul et al., 1997) to compare the amino acidic sequences of different $R$ - and $S$-IREDs previously reported (Mitsukura et al., 2010, 2013; France et al., 2018). Through the alignment of twelve sequences, conserved regions formed by 5 and 13 amino acids were identified, and from these, two were selected as templates for new searches: M1: VWNRT and M2: YxDGAI[ML]AxPx2IG. Sequenced genomes from our collection of native Streptomyces strains were analyzed using Artemis software (Carver et al., 2012), distinguishing nine sequences that presented the abovementioned motifs. These nine potential IREDs were then analyzed using additional criteria reported by Fademrecht et al. (2016) and France et al. (2017). The former characterized the presence of two protein motifs (GLGxMGx5[ATS] x4Gx4[VIL] WNR[TS $] x 2[K R]$ and Gx[DE]x[GDA]x[APS]x3 $\{\mathrm{K}\} \mathrm{x}[\mathrm{ASL}] \mathrm{x}$ [LMVIAG]) corresponding respectively to NADPH and substrate binding for IREDs. Additionally, it stablished the importance of amino acids in positions 139 and 194 for determining enzyme stereoselectivity (Fademrecht et al., 2016). Besides, we analyzed the presence of active site residues associated with reductive aminase activity (France et al., 2018). After these analyses, two potential enzymes with complementary stereoselectivity were selected: SsIRED_S from S. scabies ST129 and Ss-IRED_R from Streptomyces sp. ST1020. The selected enzymes were cloned in pET-28b $(+)$ and expressed in E. coli BL21(DE3).

\section{Characterization of Ss-IRED_S and Ss-IRED_R Biocatalytic Activity}

IRED activity for the identified enzymes was assessed using a set of substrates 1a-1d (Figure 1) encompassing both monocyclic and bulky prochiral imines. The assays were performed using resting cells under two different conditions that favor NADPH recycling: addition of glucose as co-substrate and coexpression of $\mathrm{Lb}-\mathrm{ADH}$. As can be observed in Table 1, the coexpression of ADH for NADPH recycling resulted in the best conversions as it was earlier reported by Velikogne and coworkers (Velikogne et al., 2018). Surprisingly, SsIRED_R presented no activity towards these substrates, despite the sequence alignment analysis that suggested IRED activity. On the other hand, Ss-IRED_S exhibited excellent activity towards substrates 1a-1c with superb enantiomeric excess, showing preference for bulky substrates such as those with dihydroisoquinoline and tetrahydro$\beta$-carboline motifs, and a preference for six-membered rings over fivemembered rings for monocyclic imines.

To study reductive amination activity, substrates 3-5 were tested using propargylamine (a) as amine donor (Figure 2). For these reactions, we found that recombinant $L b$-ADH reduced the ketone; thus, the assays were only performed with glucose as cosubstrate for NADPH regeneration. Following that procedure, no 
<smiles>COc1cc2c(cc1OC)C(C)=NCC2</smiles>

1a<smiles>COc1cc2c(cc1OC)[C@H](C)NCC2</smiles>

2a<smiles>CC1=NCCc2c1[nH]c1ccccc21</smiles>

$1 b$<smiles>C[C@@H]1NCCc2c1[nH]c1ccccc21</smiles>

2b<smiles>c1ccc(C2=NCCCC2)cc1</smiles>

1c<smiles>c1ccc([C@@H]2CCCCN2)cc1</smiles>

2c<smiles>c1ccc(C2=NCCC2)cc1</smiles>

1d<smiles>c1ccc([C@@H]2CCCN2)cc1</smiles>

2d

FIGURE 1 | Imines 1 and corresponding amines 2 evaluated in this study.

TABLE 1 | Characterization of the imine reductase activity: synthesis of chiral amines from the corresponding imine with Ss-IRED_S and Ss-IRED_R.

\section{Substrate}

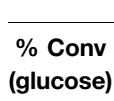

Whole cell system Ss-IRED_S

$\%$ Conv (ADH system)

$\begin{array}{lc}10 & >99 \\ 57 & >99 \\ 27 & 85 \\ <2 & <5\end{array}$

$\%$ ee

$>99(\mathrm{~S})$

$>99(\mathrm{~S})$

$98(\mathrm{R})$

nd
Whole cell system Ss-IRED R

\begin{tabular}{ccc}
\hline $\begin{array}{c}\text { Conv } \\
\text { (glucose) }\end{array}$ & $\begin{array}{c}\text { \% Conv } \\
\text { (ADH system) }\end{array}$ & $\%$ ee \\
0 & 0 & nd \\
0 & 0 & nd \\
$<1$ & $<3$ & nd \\
0 & 0 & nd
\end{tabular}<smiles>O=C1CCCCC1</smiles>

3<smiles>CC(=O)Cc1ccccc1</smiles>

4<smiles>CC(=O)CCc1ccccc1</smiles>

5<smiles>C#CCN</smiles>

a<smiles>C#CCNC1CCCCC1</smiles><smiles>C#CCN[C@@H](C)Cc1ccccc1</smiles>

$4 a$<smiles>C#CCN[C@@H](C)CCc1ccccc1</smiles>

FIGURE 2 | Ketones (3-5), propargylamine (a), and corresponding amines (3a-5a) evaluated in this study.

TABLE 2 | Characterization of the reductive amination activity: synthesis of chiral amines from the corresponding ketone and amine donor propargylamine with Ss-IRED_S and Ss-IRED_R.

\section{Substrate}

Whole cell system Ss-IRED

$\%$ Conv (Glucose)

60

0
Whole cell system Ss-IRED R

\% Conv (Glucose) \% ee

$>99$
0
40

Note. na, absolute stereochemistry could not be assigned due to lack of true optically pure standards. 
TABLE 3 | Results of in silico docking experiments with Autodock-VINA.

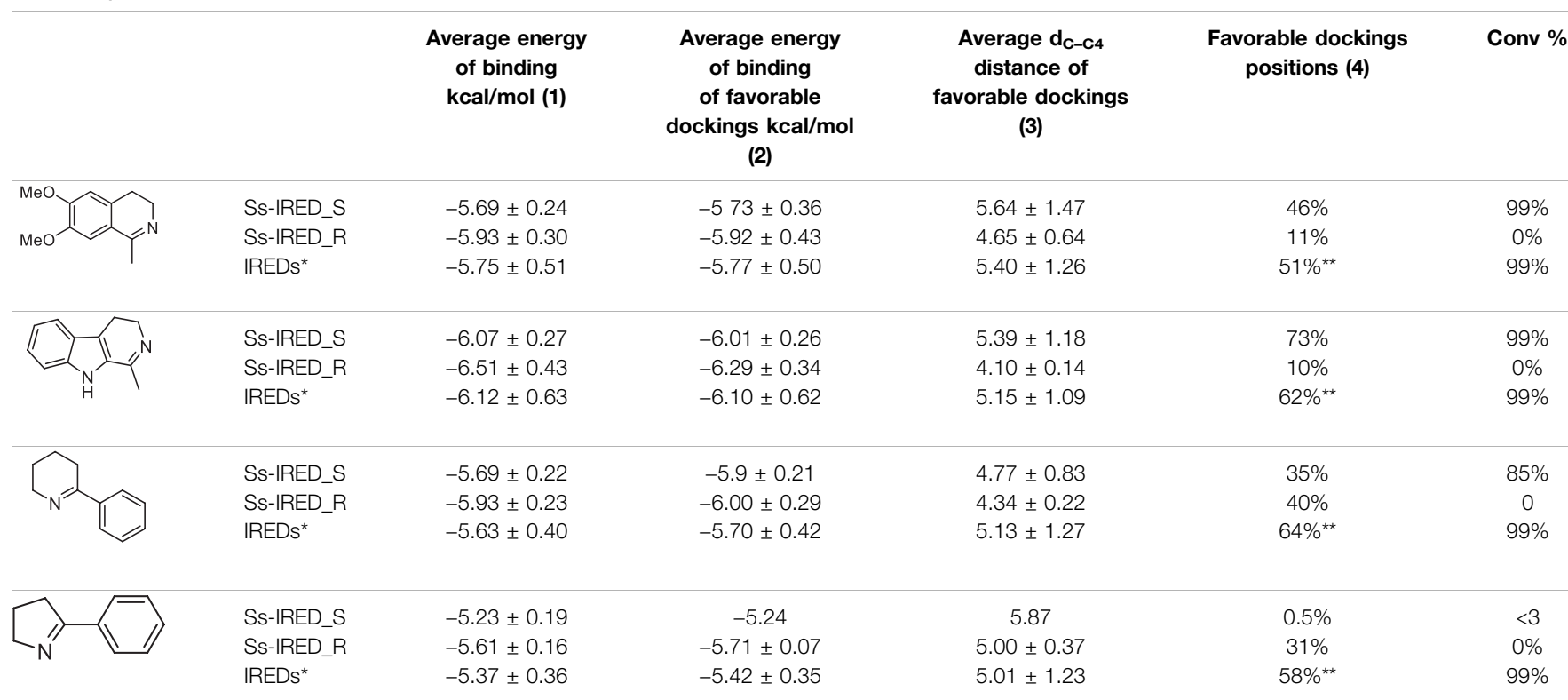

Note. (1) Average energy of binding of docked positions. (2) Energy of binding of selected positions. Distance of the imine bond and the C4 of NADPH (dC-C4), and a possible proton donor amino acid interaction were checked. (3) Average distance C-C4-NADPH of favorable selected dockings. (4) Quantitative analysis of favorable docking positions.

IREDs, imine reductases.

IREDS*: five IREDs with reported 99\% conversion used as reference; stated values are an average of the five IREDs.

ketone reduction was detected. Table 2 illustrates the experimental results obtained. As it can be observed, SsIRED_S showed moderate activity for substrate $\mathbf{3}$ and no activity with the other substrates. In contrast, Ss-IRED_R

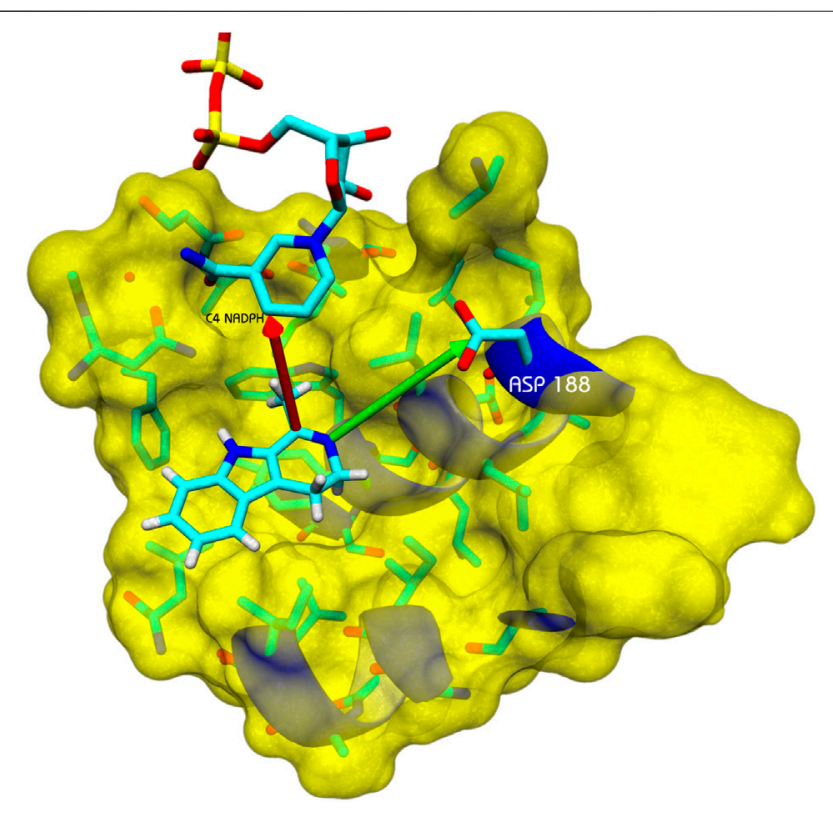

FIGURE $\mathbf{3}$ | Example of analysis of docked positions with substrate 1b. Arrow in red: distance between the $\mathrm{C} 4$ atom of the NADPH and the carbon in the $\mathrm{C}=\mathrm{N}$ double bond $\left(\mathrm{d}_{\mathrm{C}-\mathrm{C} 4}\right)$. Arrow in green: distance of an acidic amino acid to act as a proton donor or as part of an $\mathrm{H}$-bonding network. presented good reductive amination activity with substrates 3 and 5, this last one with excellent enantiomeric excess. Despite many efforts on assaying the reductive amination of phenyl ketone (4), no activity was detected for this substrate.

\section{Homology Models and Docking Analysis}

Imine reduction and reductive amination have been observed with highly diverse sequences, which indicates the importance of analyzing the three-dimensional structure and the characteristics of the active site over that of the linear sequence. In silico analysis was performed for the new enzymes to better understand observed preferences for IRED or RedAm activities, as well as differences in substrate specificity.

Homology models for Ss-IRED_R (Supplementary Figure S1), Ss-IRED_S (Supplementary Figure S2), and 10 IREDs used as controls in complex with NADPH cofactor were constructed using the "closed" form with NADPH of IREDs in PDB because analysis of the (apo-) and (NADPH-bound) subunits within the structure revealed a rotation of $14^{\circ}$ (Sharma et al., 2018). Docking studies of substrates 1a-1d and 3-5 and a in the different enzymes' active site were performed by Autodock-VINA. After clustering of 250 runs of each substrate with each enzyme, the different ligand-protein interactions in the active site were analyzed (Figure 3). To analyze IRED activity, substrates 1a-1d in the different enzymes were selected for docking, and different parameters such as energy of binding, distance for hydride transfer from $\mathrm{NADPH}$ to $\mathrm{C}=\mathrm{N}$ double bond, and distance between nitrogen of imine with surrounding acidic amino acids were evaluated. Adequate distance between the $\mathrm{C} 4$ atom of the NADPH and the carbon in the $\mathrm{C}=\mathrm{N}$ double bond $\left(\mathrm{d}_{\mathrm{C}-\mathrm{C} 4}\right)$ is needed for hydride delivery. 
Additionally, mechanistic studies have suggested the importance of an acidic amino acid at a proper distance to the forming amine to act directly or through a water molecule as a proton donor (Man et al., 2015).

Favorable docking positions were selected as those presenting a $\mathrm{d}_{\mathrm{C}-\mathrm{C} 4}$ distance minor to $7.5 \AA$, and a possible proton donor amino acid at a distance minor to $7 \AA$. Previous reports have indicated Tyr 169 in GF3546-IRED from Streptomyces sp. or Asp 187 in Q1EQE0 IRED from Streptomyces kanamyceticus as candidates for protonating the forming amine (Rodríguez-Mata et al., 2013; Man et al., 2015). The presence of these residues was first analyzed, but the presence of other acidic amino acids at an adequate distance was equally considered to support catalytic activity (Ribeiro et al., 2020). A similar analysis was performed on a group of five IREDs (IREDs ${ }^{\star}$ ) whose activity was previously reported using the same substrates in order to compare the different parameters (Velikogne et al., 2018). The results are presented in Table 3.

As can be seen in Table 3, the results of this in silico study indicate that no significant difference in energy of binding is detected when considering all the docked positions or only favorable ones, despite evidence of excellent activity associated with these enzymes (columns 1 and 2 ). Regarding the $\mathrm{d}_{\mathrm{C}-\mathrm{C} 4}$ distance, it could be observed that selected positions show an average value of $5 \AA$, as well as those for the control group. Surprisingly, Ss-IRED_R presented the shortest average distance ( $\sim 4.5 \AA$ ) with all substrates even though no IRED activity was detected with this enzyme. A correlation between $\mathrm{d}_{\mathrm{C}-\mathrm{C} 4}$ distance and activity cannot be clearly established from these data.

Interestingly, the percentage of docked positions complying with catalytic requirements correlates well with experimental activity. A quantitative analysis of docking positions for substrates $\mathbf{1 a}$ and $\mathbf{1 b}$ in Ss-IRED_S yields respectively $46 \%$ and $73 \%$ of favorable positions that meet the catalytic requirements. It is noteworthy that a similar quantitative percentage of positions is observed for the IREDs in control group. Concurrently, for the inactive Ss-IRED_R, only 10\% of the positions of substrate $\mathbf{1 a}$ and $11 \%$ of $\mathbf{1 b}$ comply with the expected attributes for catalysis. Additionally, for substrate 1d, IREDs in the control group show an average of $58 \%$ of positions presenting an adequate distance to NADPH and the required amino acid for amine protonation. In agreement with these data, Ss-IRED_S and SsIRED_R with no detectable activity presented respectively only $0.5 \%$ and $31 \%$ of positions complying with catalytic requirements. The results for substrate $\mathbf{1 c}$ are inconsistent at first sight; however, a deeper analysis allowed us to determine that for the inactive SsIRED_R, most docked positions in clusters do not interact with Asp176 but with a different possible proton donor. On the contrary, Ss-IRED_S presents interaction with Asp188 in most positions in the selected clusters, which could explain the difference in activity (Supplementary Table S3).

A similar docking analysis to explain reductive aminase results was investigated; however, no correlation could be established in this case (data not shown). Therefore, we based our assessment of Ss-IRED_S and Ss-IRED_R activity on the work reported by France et al. (2017). The presence of the described critical conserved amino acids in the active site was examined based on an alignment of our enzymes with the best reductive aminases reported by these authors (Supplementary Table S7). We noticed that Ss-IRED_R presents the three amino acids that interact directly with the main functional groups of our substrates via hydrogen bonding, while it also presents the other three conserved amino acids to which no specific function has been assigned. In contrast, Ss-IRED_S only has two of the three conserved amino acids that interact directly with the substrates.

\section{DISCUSSION}

Two new IREDs, Ss-IRED_S and Ss-IRED_R, have been identified from native Streptomyces strains isolated from Uruguayan soil. The strategy followed for identification was based on the conserved motifs M1: VWNRT and M2: YxDGAI[ML]AxPx2IG and proved successful since both enzymes presented IRED or reductive aminase activity. This approach could yield more diverse enzymes than procedures based strictly on BLAST or homology search, overall when imine reduction and reductive amination have been observed with highly diverse sequences, indicating the importance of threedimensional structure and the characteristics of the active site (Marshall et al., 2020; Montgomery et al., 2020). A feature that is observed in these structures is their highly conserved NADPH binding site. When the stereoselectivity is analyzed in Ss-IRED_S and Ss-IRED_R, prediction by the presence of key residues identified by Fademrecht and co-workers seems to work well (Fademrecht et al., 2016,) although the origin of IRED selectivity is complex and not fully understood.

A recent study by Velikogne and co-workers classifies a selection of published and novel IREDs as either "D-type" or "Y-type" based on phylogenetic tree analysis and whether they have an aspartic acid or a tyrosine residue aligned with position 187 in S. kanamyceticus Q1EQE0 IRED (Velikogne et al., 2018). The catalytic profile of D-type enzymes was characterized with several substrates, presenting particularly poor results with substrates $\mathbf{1 a}$ and $\mathbf{1 b}$. A detail to remark from our results is that both of our IREDs are D-types enzymes; however, our Ss-IRED_S present excellent conversion with sterically demanding imines $\mathbf{1 a}$ and $\mathbf{1 b}$ (Table 1). Additionally, our enzymes presented poor activity with $\mathbf{1 d}$, which proved to be a good substrate for most D-type enzymes. Considering both substrate specificity and stereoselectivity, Ss-IRED_S behaves like a Y-type enzyme. Although the association of structural data with enzyme activity is really important, it has remained elusive for IRED activity, overall when based in one amino acid and exceptions are found.

The results presented in Table 1 clearly demonstrate that coexpression of a cofactor regeneration system for NADPH recycling is essential for good IRED activity with whole cell biocatalysts. $\mathrm{ADH}$-based system reported by Velikogne et al. is extremely efficient for imine reduction (Velikogne et al., 2018); however, its own biocatalytic activity hampered its use for reductive aminations since it reduced the ketone. It would be highly desirable to devise a similar system for NADPH recycling that avoids ketone reduction.

Various authors reported excellent results for reductive amination with purified enzymes (Aleku et al., 2017; Matzel, Gand, and Höhne 2017; Ramsden et al., 2019; Yao et al., 2021); 
however, only one previous work focused on the applicability of whole cell biocatalysts for this reaction (Maugeri and Rother 2017). In a screening of 15 IRED systems, Maugeri et al. reported only one system with $99 \%$ of conversion with cyclohexanone as a substrate. Table 2 shows that the whole cell system expressing SsIRED_R can be used for reductive aminations with excellent results, and no ketone reduction by $E$. coli native dehydrogenases was detected. The reductive amination of phenylacetone (4) and 4-phenyl-2-butanone (5) with propargylamine (a) has proven a challenging transformation even for isolated RedAm, and few literature reports are available (Aleku et al., 2017; Marshall et al., 2020). The activity and stereoselectivity presented by Ss-IRED_R with substrate $\mathbf{5}$ situate this enzyme as a good platform for targeted evolution. The absence of activity with substrate $\mathbf{4}$ is intriguing; however, the conjugated $\pi$-system in this substrate could favor the enolate form, which could be further stabilized by residues in the active site impeding reductive amination due to steric or electronic issues.

With the aim of contributing to the analysis of possible correlations among catalytic activity and structural features, we considered performing an in silico analysis of our enzymes compared with previously reported ones. Among the available computational approaches, we chose to perform homology modeling and docking analysis, since it does not demand high levels of computing power and could be used to explain experimental data. The docking studies performed in this work confirmed the complexity of the active sites of enzymes with IRED-RedAm activities. Considering the energy of binding, values obtained with Autodock-VINA are not enough to ensure the activity of an enzyme with a tested substrate, and a deeper analysis using molecular dynamics and free energy calculations could provide a better though more time-consuming approach. Furthermore, the values obtained with the applied methodology presented a large dispersion, and no significant correlation between catalytic activity and energy of binding could be recognized. Nevertheless, it is worth noticing that when other restrictions are applied, such as the catalytic requirements used here (distance $\mathrm{C}-\mathrm{C}_{4}$ and interaction with proton donor amino acids interaction of nitrogen of imine), the results obtained seem to correlate with the observed catalytic activity.

Even though IRED's active site residues show significant variation, making it challenging to clearly define the characteristics of this enzyme family or to predict the properties of uncharacterized homologues, it is undeniable that residues structurally aligned to positions 169 or 187 play a key role either as proton donors or as anchors of the substrate in an optimal position. Experimental and crystallographic data showed that residue Asp187 in SkIRED (IRED-R type) or Tyr169 in BcIRED and NhIRED (IRED-S type) played an important role in activity, since mutation of these residues resulted in an inactive enzyme (Rodríguez-Mata et al., 2013; Man et al., 2015). Nevertheless, some discrepancy arose from other studies in which similar enzyme mutants maintain relative activity (Scheller et al., 2014; Hussain et al., 2015; Aleku et al., 2017); however, it should be noted that other residues can also serve as proton donors allowing catalysis (Aleku et al., 2016). In our in silico analysis, we have considered both possibilities, and the presence of an acidic amino acid at an adequate distance for proton transfer was established as a catalytic requirement. This criterion, taken together with the evaluation of the distance for hydride transfer from $\mathrm{NADPH}$ to $\mathrm{C}=\mathrm{N}$ double bond, proved to correlate well in most cases with biocatalytic activity.

As a summary, the use of bioinformatic analysis based on sequence and structural features proved useful for the identification of novel IREDs. Furthermore, the criteria established by Fademrecht et al. remain interesting for prediction of stereoselectivity, and the evaluation of amino acids in the active site proposed by France et al. correlates well for predicting RedAm activity. The application of these criteria helped us in selecting two IRED candidates from native Streptomyces genomes. The described Ss-IRED-S constitutes an interesting addition to the biocatalytic toolbox due to its excellent activity and enantioselectivity for the reduction of bulky imines, while Ss-IRED-R adds to the repertoire of reductive aminases. Modeling each enzyme candidate and performing docking studies as those presented in this work could help in predicting substrate specificity. This proved useful for IREDs; however, due to a more complex mechanism (Sharma et al., 2018), additional work should be done in order to establish a similar procedure for reductive amination activity. Despite this, our results show that considering the percent of docking positions complying with catalytic requirements could be a useful new addition to the in silico search for novel enzymes with IRED activity, avoiding testing of large enzyme libraries and facilitating prediction of enzyme activity.

\section{DATA AVAILABILITY STATEMENT}

The original contributions presented in the study are included in the article/Supplementary Materials. Further inquiries can be directed to the corresponding authors.

\section{AUTHOR CONTRIBUTIONS}

CI performed most of the bioinformatic analysis, homology modeling and docking studies, and analysis of correlation among bioinformatic and experimental data. AT performed work on Artemis for IRED identification, and GL and PP deepened the analysis by performing and analyzing alignments with other enzymes. AT and GL equally contributed with characterization of Ss-IRED_S and Ss-IRED_R by performing cloning, expression, and biotransformation experiments. Both developed analytical conditions for GC analysis of the different compounds. ML isolated Streptomyces genomes and guided work with Artemis software. MP constructed and provided the Streptomyces culture collection. GL performed Ss-IRED_R modeling and docking studies to explain RedAm activity guided by CI and PP. CI, PP, and SR made substantial contributions to the conception and design of the research; however, all authors were involved in the acquisition, analysis, and interpretation of data used in the work. CI, AT, PP, and SR 
had a major contribution on writing the present manuscript; GL has also been involved in manuscript preparation, critical revision, and approval of the final version. All the authors agree to be accountable for all aspects of the work in ensuring that questions related to the accuracy or integrity of any part of the work are appropriately investigated and resolved.

\section{FUNDING}

Funding for lab reagents and supplies was from Programa de Desarrollo de las Ciencias Básicas (PEDECIBA); Centro CEIBOS Espacio Interdisciplinario, Udelar; CSIC, Udelar (CSIC I +D 1515-602 and CSIC I+D 2129-347); ANII FMV_1_2017_1_136610 and L'Oreal-UNESCO For Women in Science, Uruguay. Funding for scholarships was from ANII POS_NAC_M_2020_1_164289 and CAP-Udelar-2015.

\section{REFERENCES}

Adams, J. P., Brown, M. J. B., Diaz-Rodriguez, A., Lloyd, R. C., and Roiban, G. D. (2019). Biocatalysis: A Pharma Perspective. Adv. Synth. Catal. 361, 2421-2432. doi:10.1002/adsc.201900424

Aleku, G. A., France, S. P., Man, H., Mangas-Sanchez, J., Montgomery, S. L., Sharma, M., et al. (2017). A Reductive Aminase from Aspergillus Oryzae. Nat. Chem 9, 961-969. doi:10.1038/nchem.2782

Aleku, G. A., Man, H., France, S. P., Leipold, F., Hussain, S., Toca-Gonzalez, L., et al. (2016). Stereoselectivity and Structural Characterization of an Imine Reductase (IRED) from Amycolatopsis Orientalis. ACS Catal. 6, 3880-3889. doi:10.1021/acscatal.6b00782

Altschul, S., Madden, T. L., Schäffer, A. A., Zhang, J., Zhang, Z., Miller, W., et al. (1997). Gapped BLAST and PSI-BLAST: A New Generation of Protein Database Search Programs. Nucleic Acids Res. 25, 3389-3402. doi:10.1093/nar/25.17.3389

Bommarius, A. S., Blum, J. K., and Abrahamson, M. J. (2011). Status of Protein Engineering for Biocatalysts: How to Design an Industrially Useful Biocatalyst. Curr. Opin. Chem. Biol. 15, 194-200. doi:10.1016/j.cbpa.2010.11.011

Carver, T., Harris, S. R., Berriman, M., Parkhill, J., and McQuillan, J. A. (2012). Artemis: An Integrated Platform for Visualization and Analysis of HighThroughput Sequence-Based Experimental Data. Bioinformatics 28, 464-469. doi:10.1093/bioinformatics/btr703

Catucci, G., Romagnolo, A., Spina, F., Varese, G. C., Gilardi, G., and Di Nardo, G. (2016). Enzyme-Substrate Matching in Biocatalysis: In Silico Studies to Predict Substrate Preference of Ten Putative Ene-Reductases from Mucor Circinelloides MUT44. J. Mol. Catal. B: Enzymatic 131, 94-100. doi:10.1016/j.molcatb.2016.06.008

Cosgrove, S. C., Brzezniak, A., France, S. P., Ramsden, J. I., Mangas-Sanchez, J., Montgomery, S. L., et al. (2018). Imine Reductases, Reductive Aminases, and Amine Oxidases for the Synthesis of Chiral Amines: Discovery, Characterization, and Synthetic Applications. Methods Enzymol. 608, 131-149. doi:10.1016/bs.mie.2018.04.022

Croce, V., López-Radcenco, A., Lapaz, M. I., Pianzzola, M. J., Moyna, G., and Siri, M. I. (2021). An Integrative Approach for the Characterization of PlantPathogenic Streptomyces Spp. Strains Based on Metabolomic, Bioactivity, and Phylogenetic Analysis. Front. Microbiol. 12, 1-11. doi:10.3389/ fmicb.2021.643792

Devine, P. N., Howard, R. M., Kumar, R., Thompson, M. P., Truppo, M. D., and Turner, N. J. (2018). Extending the Application of Biocatalysis to Meet the Challenges of Drug Development. Nat. Rev. Chem. 2, 409-421. doi:10.1038/ s41570-018-0055-1

Duan, S., Widlicka, D. W., Burns, M. P., Kumar, R., Hotham, I., Desrosiers, J.-N., et al. (2021). Application of Biocatalytic Reductive Amination for the Synthesis of a Key Intermediate to a CDK 2/4/6 Inhibitor. Org. Process. Res. Dev. doi:10.1021/acs.oprd.1c00255

\section{ACKNOWLEDGMENTS}

The authors wish to thank Jörg H. Schrittwieser for providing plasmid pASK-IBA5plus $-L b$-ADH for NADPH regeneration along with pET-28a(+)-based expression vectors for IRED-A to IRED-N used as controls in different experiments; Nicholas Turner for kindly supplying substrates $\mathbf{1 b}-\mathbf{1 d}$ as well as pET 28a-His-AspRedAm plasmid used as control for reductive amination reactions; and Ignacio Carrera for offering substrate 1a. Additionally, SR wishes to thank Carrera for his support and for thoughtful discussions.

\section{SUPPLEMENTARY MATERIAL}

The Supplementary Material for this article can be found online at: https://www.frontiersin.org/articles/10.3389/fctls.2021.785963/ full\#supplementary-material

Fademrecht, S., Scheller, P. N., Nestl, B. M., Hauer, B., and Pleiss, J. (2016). Identification of Imine Reductase-specific Sequence Motifs. Proteins 84, 600-610. doi:10.1002/prot.25008

France, S. P., Aleku, G. A., Sharma, M., Mangas-Sanchez, J., Howard, R. M., Steflik, J., et al. (2017). Biocatalytic Routes to Enantiomerically Enriched Dibenz [c ,e ]azepines. Angew. Chem. Int. Ed. 56, 15589-15593. doi:10.1002/ anie. 201708453

France, S. P., Howard, R. M., Steflik, J., Weise, N. J., Mangas-Sanchez, J., Montgomery, S. L., et al. (2018). Identification of Novel Bacterial Members of the Imine Reductase Enzyme Family that Perform Reductive Amination. ChemCatChem 10, 510-514. doi:10.1002/cctc.201701408

Han, S.-W., Kim, J., Cho, H.-S., and Shin, J.-S. (2017). Active Site Engineering of $\omega$-Transaminase Guided by Docking Orientation Analysis and Virtual Activity Screening. ACS Catal. 7, 3752-3762. doi:10.1021/acscatal.6b03242

Höhne, M. (2019). Engineering Imine Reductases for Industrial Applications. Nat. Catal. 2, 841-842. doi:10.1038/s41929-019-0366-8

Höhne, M., Schätzle, S., Jochens, H., Robins, K., and Bornscheuer, U. T. (2010). Rational Assignment of Key Motifs for Function Guides In Silico Enzyme Identification. Nat. Chem. Biol. 6, 807-813. doi:10.1038/nchembio.447

Hussain, S., Leipold, F., Man, H., Wells, E., France, S. P., Mulholland, K. R., et al. (2015). An (R)-Imine Reductase Biocatalyst for the Asymmetric Reduction of Cyclic Imines. ChemCatChem 7, 579-583. doi:10.1002/cctc.201402797

Iglesias, C., Panizza, P., and Rodriguez Giordano, S. (2017). Identification, Expression and Characterization of an R- $\omega$-Transaminase from Capronia Semiimmersa. Appl. Microbiol. Biotechnol. 101, 5677-5687. doi:10.1007/ s00253-017-8309-2

Jones, D. T. (1999). Protein Secondary Structure Prediction Based on Positionspecific Scoring Matrices 1 1Edited by G. Von Heijne. J. Mol. Biol. 292, 195-202. doi:10.1006/jmbi.1999.3091

Kohls, H., Steffen-Munsberg, F., and Höhne, M. (2014). Recent Achievements in Developing the Biocatalytic Toolbox for Chiral Amine Synthesis. Curr. Opin. Chem. Biol. 19, 180-192. doi:10.1016/j.cbpa.2014.02.021

Krieger, E., Darden, T., Nabuurs, S. B., Finkelstein, A., and Vriend, G. (2004). Making Optimal Use of Empirical Energy Functions: Force-Field Parameterization in Crystal Space. Proteins 57, 678-683. doi:10.1002/ prot.20251

Krieger, E., Koraimann, G., and Vriend, G. (2002). Increasing the Precision of Comparative Models with YASARA NOVA-a Self-Parameterizing Force Field. Proteins 47, 393-402. doi:10.1002/prot.10104

Krieger, E., Dunbrack Jr, R. L., Hooft, R. W., and Krieger, B. (2012). Assignment of Protonation States in Proteins and Ligands: Combining pKa Prediction with Hydrogen Bonding Network Optimization. Methods Mol. Biol. 819, 405-421. doi:10.1007/978-1-61779-465-0_25

Krieger, E., and Vriend, G. (2014). YASARA View-Molecular Graphics for All Devices-From Smartphones to Workstations. Bioinformatics (Oxford, England) 30, 2981-2982. doi:10.1093/bioinformatics/btu426 
Kumar, R., Karmilowicz, M. J., Burke, D., Burns, M. P., Clark, L. A., Connor, C. G., et al. (2021). Biocatalytic Reductive Amination from Discovery to Commercial Manufacturing Applied to Abrocitinib JAK1 Inhibitor. Nat. Catal. 4, 775-782. doi:10.1038/s41929-021-00671-5

Lapaz, M. I., Huguet-Tapia, J. C., Siri, M. I., Verdier, E., Loria, R., and Pianzzola, M. J. (2017). Genotypic and Phenotypic Characterization of Streptomyces Species Causing Potato Common Scab in Uruguay. Plant Dis. 101, 1362-1372. doi:10.1094/PDIS-09-16-1348-RE

Lapaz, M. I., López, A., Huguet-Tapia, J. C., Pérez-Baldassari, M. F., Iglesias, C., Loria, R., et al. (2019). Isolation and Structural Characterization of a Nondiketopiperazine Phytotoxin from a Potato Pathogenic Streptomyces Strain. Nat. Product. Res. 33, 2951-2957. doi:10.1080/14786419.2018.1511554

Lenz, M., Borlinghaus, N., Weinmann, L., and Nestl, B. M. (2017). Recent Advances in Imine Reductase-Catalyzed Reactions. World J. Microbiol. Biotechnol. 33, 10. doi:10.1007/s11274-017-2365-8

Man, H., Wells, E., Hussain, S., Leipold, F., Hart, S., Turkenburg, J. P., et al. (2015). Structure, Activity and Stereoselectivity of NADPH-dependent Oxidoreductases Catalysing theS-Selective Reduction of the Imine Substrate 2-Methylpyrroline. ChemBioChem 16, 1052-1059. doi:10.1002/cbic.201402625

Marshall, J. R., Yao, P., Montgomery, S. L., Finnigan, J. D., Thorpe, T. W., Palmer, R. B., et al. (2020). Screening and Characterization of a Diverse Panel of Metagenomic Imine Reductases for Biocatalytic Reductive Amination. Nat. Chem. 13, 140-148. doi:10.1038/s41557-020-00606-w

Matzel, P., Gand, M., and Höhne, M. (2017). One-Step Asymmetric Synthesis of (R)- and (S)-Rasagiline by Reductive Amination Applying Imine Reductases. Green. Chem. 19, 385-389. doi:10.1039/c6gc03023h

Maugeri, Z., and Rother, D. (2017). Reductive Amination of Ketones Catalyzed by Whole Cell Biocatalysts Containing Imine Reductases (IREDs). J. Biotechnol. 258, 167-170. doi:10.1016/j.jbiotec.2017.05.015

Mitsukura, K., Kuramoto, T., Yoshida, T., Kimoto, N., Yamamoto, H., and Nagasawa, T. (2013). A NADPH-dependent (S)-Imine Reductase (SIR) from Streptomyces Sp. GF3546 for Asymmetric Synthesis of Optically Active Amines: Purification, Characterization, Gene Cloning, and Expression. Appl. Microbiol. Biotechnol. 97, 8079-8086. doi:10.1007/s00253-012-4629-4

Mitsukura, K., Suzuki, M., Tada, K., Yoshida, T., and Nagasawa, T. (2010). Asymmetric Synthesis of Chiral Cyclic Amine from Cyclic Imine by Bacterial Whole-Cell Catalyst of Enantioselective Imine Reductase. Org. Biomol. Chem. 8, 4533-4535. doi:10.1039/c0ob00353k

Mitsukura, K., Suzuki, M., Shinoda, S., Kuramoto, T., Yoshida, T., and Nagasawa, T. (2011). Purification and Characterization of a Novel (R)-Imine Reductase from Streptomyces ssp. GF3587. Biosci. Biotechnol. Biochem. 75, 1778-1782. doi:10.1271/bbb.110303

Montgomery, S. L., Pushpanath, A., Heath, R. S., Marshall, J. R., Klemstein, U., Galman, J. L., et al. (2020). Characterization of Imine Reductases in Reductive Amination for the Exploration of Structure-Activity Relationships. Sci. Adv. 6, eaay9320. doi:10.1126/sciadv.aay 9320

Pronobis, M. I., Deuitch, N., and Peifer, M. (2016). The Miraprep: A Protocol that Uses a Miniprep Kit and Provides Maxiprep Yields. PLoS ONE 11, e0160509-12. doi:10.1371/journal.pone.0160509

Ramsden, J. I., Heath, R. S., Derrington, S. R., Montgomery, S. L., Mangas-Sanchez, J., Mulholland, K. R., et al. (2019). Biocatalytic N-Alkylation of Amines Using Either Primary Alcohols or Carboxylic Acids via Reductive Aminase Cascades. J. Am. Chem. Soc. 141, 1201-1206. doi:10.1021/jacs.8b11561

Ribeiro, A. J. M., Tyzack, J. D., Borkakoti, N., Holliday, G. L., and Thornton, J. M. (2020). A Global Analysis of Function and Conservation of Catalytic Residues in Enzymes. J. Biol. Chem. 295, 314-324. doi:10.1074/jbc.REV119.006289

Rinaldi, S., Van Der Kamp, M. W., Ranaghan, K. E., Mulholland, A. J., and Colombo, G. (2018). Understanding Complex Mechanisms of Enzyme Reactivity: The Case of Limonene-1,2-Epoxide Hydrolases. ACS Catal. 8, 5698-5707. doi:10.1021/acscatal.8b00863

Rodríguez-Mata, M., Frank, A., Wells, E., Leipold, F., Turner, N. J., Hart, S., et al. (2013). Structure and Activity of NADPH-dependent Reductase Q1EQE0 fromStreptomyces Kanamyceticus, Which Catalyses theR-Selective Reduction of an Imine Substrate. ChemBioChem 14, 1372-1379. doi:10.1002/cbic.201300321

Sambrook, J. (2001). Molecular Cloning : A Laboratory Manual. Cold Spring Harbor, N.Y: Cold Spring Harbor Laboratory Press.
Scheller, P. N., Fademrecht, S., Hofelzer, S., Pleiss, J., Leipold, F., Turner, N. J., et al. (2014). Enzyme Toolbox: Novel Enantiocomplementary Imine Reductases. ChemBioChem 15, 2201-2204. doi:10.1002/cbic.201402213

Schober, M., MacDermaid, C., Ollis, A. A., Chang, S., Khan, D., Hosford, J., et al. (2019). Chiral Synthesis of LSD1 Inhibitor GSK2879552 Enabled by Directed Evolution of an Imine Reductase. Nat. Catal. 2, 909-915. doi:10.1038/s41929019-0341-4

Schrittwieser, J. H., Velikogne, S., and Kroutil, W. (2015). Biocatalytic Imine Reduction and Reductive Amination of Ketones. Adv. Synth. Catal. 357, 1655-1685. doi:10.1002/adsc.201500213

Senior, A. W., Evans, R., Jumper, J., Kirkpatrick, J., Sifre, L., Green, T., et al. (2020). Improved Protein Structure Prediction Using Potentials from Deep Learning. Nature 577, 706-710. doi:10.1038/s41586-019-1923-7

Senior, A. W., Evans, R., Jumper, J., Kirkpatrick, J., Sifre, L., Green, T., et al. (2019). Protein Structure Prediction Using Multiple Deep Neural Networks in the 13th Critical Assessment of Protein Structure Prediction (CASP13). Proteins 87, 1141-1148. doi:10.1002/prot.25834

Sharma, M., Mangas-Sanchez, J., France, S. P., Aleku, G. A., Montgomery, S. L., Ramsden, J. I., et al. (2018). A Mechanism for Reductive Amination Catalyzed by Fungal Reductive Aminases. ACS Catal. 8, 11534-11541. doi:10.1021/ acscatal.8b03491

Siddappa, N. B., Avinash, A., Venkatramanan, M., and Ranga., U. (2007). Regeneration of Commercial Nucleic Acid Extraction Columns without the Risk of Carryover Contamination. BioTechniques 42, 186-192. doi:10.2144/ 000112327

Sirin, S., Kumar, R., Martinez, C., Karmilowicz, M. J., Ghosh, P., Abramov, Y. A., et al. (2014). A Computational Approach to Enzyme Design: Predicting $\omega$-Aminotransferase Catalytic Activity Using Docking and MM-GBSA Scoring. J. Chem. Inf. Model. 54, 2334-2346. doi:10.1021/ci5002185

Spasic, J., Mandic, M., Djokic, L., Djokic, L., and Nikodinovic-Runic, J. (2018). Streptomyces Spp. In the Biocatalysis Toolbox. Appl. Microbiol. Biotechnol. 102, 3513-3536. doi:10.1007/s00253-018-8884-x

Trott, O., and Olson, A. J. (2019). AutoDock Vina: Improving the Speed and Accuracy of Docking with a New Scoring Function, Efficient Optimization, and Multithreading. J. Comput. Chem. 31, 455-461. doi:10.1002/jcc.21334.AutoDock

Velikogne, S., Resch, V., Dertnig, C., Schrittwieser, J. H., and Kroutil, W. (2018). Sequence-Based In-silico Discovery, Characterisation, and Biocatalytic Application of a Set of Imine Reductases. ChemCatChem 10, 3236-3246. doi: $10.1002 /$ cctc. 201800607

Wetzl, D., Gand, M., Ross, A., Müller, H., Matzel, P., Hanlon, S. P., et al. (2016). Asymmetric Reductive Amination of Ketones Catalyzed by Imine Reductases. ChemCatChem 8, 2023-2026. doi:10.1002/cctc.201600384

Wood, W. N., Smith, K. D., Ream, J. A., and Lewis, L. K. (2017). Enhancing Yields of Low and Single Copy Number Plasmid DNAs from Escherichia Coli Cells. J. Microbiol. Methods 133, 46-51. doi:10.1016/j.mimet.2016.12.016

Yao, P., Marshall, J. R., Xu, Z., Lim, J., Charnock, S. J., Zhu, D., et al. (2021). Asymmetric Synthesis of N -Substituted a-Amino Esters from $\alpha$-Ketoesters via Imine Reductase-Catalyzed Reductive Amination. Angew. Chem. Int. Ed. 60, 8717-8721. doi:10.1002/anie.202016589

Conflict of Interest: The authors declare that the research was conducted in the absence of any commercial or financial relationships that could be construed as a potential conflict of interest.

Publisher's Note: All claims expressed in this article are solely those of the authors and do not necessarily represent those of their affiliated organizations, or those of the publisher, the editors, and the reviewers. Any product that may be evaluated in this article, or claim that may be made by its manufacturer, is not guaranteed or endorsed by the publisher.

Copyright () 2021 Iglesias, Tijman, López, Lapaz, Pianzzola, Panizza and Rodríguez Giordano. This is an open-access article distributed under the terms of the Creative Commons Attribution License (CC BY). The use, distribution or reproduction in other forums is permitted, provided the original author(s) and the copyright owner(s) are credited and that the original publication in this journal is cited, in accordance with accepted academic practice. No use, distribution or reproduction is permitted which does not comply with these terms. 